\title{
Response of Tomato Plants to Irrigation with Magnetized Water and some Foliar Application Treatments under Drip Irrigation System: 1-Vegetative Growth and Chemical Constituents of Leaves. Dawa, K. K.; H. M. E. Abd El-Nabi and W. M. E. Swelam Veget. and Flori. Dept. Fac. Agric., Mans. Univ., Egypt.
}

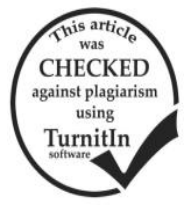

\section{ABSTRACT}

This experiment was conducted in summer seasons of 2016 and 2017 on tomato plants "6112" hybrid to evaluate tomato plants performance (vegetative growth characteristics and chemical constituents of leaves) in response to irrigation water treatments (magnetized and non-magnetized), foliar applications (chitosan, lithovit, selenium and yeast extract) and their interactions. Results indicated that the highest values of vegetative growth characteristics, i.e., plant height, number of branches, number of leaves, leaf area, fresh and dry weights as well as chemical constituents of leaves as photosynthetic pigments (Chl. a, Chl. b, total Chl. a+b and carotenoids) and leaf minerals content $(\mathrm{N}, \mathrm{P}, \mathrm{K}, \mathrm{Ca}, \mathrm{Mg}, \mathrm{Zn}, \mathrm{Mn})$ were recorded when plants irrigated with magnetized water as compared with plants irrigated with untreated water in both studying seasons. On the other hand, Fe content responded negatively to irrigation with magnetized water.Comparing the effect of foliar applications, all foliar application treatments significantly enhanced vegetative growth parameters, leaf minerals and pigments contents compared to the check treatment. Foliar application of chitosan at 250 ppm is the superior in its effect on all the aforementioned characteristics followed by yeast extract at $10 \mathrm{~g} / \mathrm{L}$ then lithovit at $1.5 \mathrm{~g} / \mathrm{L}$ in the two seasons except for Fe content. The best results of both vegetative growth attributes and chemical constituents of leaves were recorded when plants irrigated with magnetized water and sprayed with chitosan at $250 \mathrm{ppm}$ in both seasons. Thus, this treatment could be recommended to improve tomato plants performance under similar conditions of this study.

Keywords: tomato plants, foliar application, chitosan, lithovit, selenium, yeast extract, vegetative growth, chemical composition.

\section{INTRODUCTION}

Tomato (Lycopersicon esculentum Mill), belongs to the family Solanaceae. Most of fruits are sold fresh, but large amounts are also processed. Fruits are used as a fresh salad vegetable, in stews, sauces, soups and other various dishes. Tomato fruits are consumed like a functional food all over the world due to the health promoting compounds and the different antioxidant molecules such as carotenoids, ascorbic acid, vitamin $\mathrm{E}$ and polyphenol compounds such as flavonoids. Tomatoes also contain minerals such as calcium, magnesium, iron and potassium, as well as microelements such a copper, zinc and manganese in addition to selenium.

There are previous studies indicated that magnetic treatment of irrigation water offers many benefits in agriculture such as enhancing vegetative growth, increasing yield, early maturity of crops, improving crop quality, increasing fertilizers efficiency and reducing cost of farm operations (Maheshwari and Grewal, 2009).

Using bio stimulants (chitosan and yeast extract) to promote plant growth has recently gained increasing attention worldwide. Chitosan a co-polymer of N-acetyl-dglucosamine and d-glucosamine, formed from chitin, and applied in horticulture as a bio stimulant to induce pathogen resistance, enhance plant growth and abiotic stress tolerance and to. some researchers reported that chitosan enhanced plants performance, thereby increasing key enzymes activities of nitrogen metabolism (nitrate reductase, glutamine synthetase) and improving transportation of nitrogen $(\mathrm{N})$ in leaves which in turn enhanced plant growth and development (Chibu and Shibayama, 2003).

Selenium is a trace element essential for both animals and plants, but is toxic at higher concentrations. Selenium content in soils varies considerably, and its availability in agricultural soils is usually low, therefore, Se is often used in fertilizers for crops. Several researchers have described the effect of Se application on vegetable crops (Abul-Soud and Abd Elrahman, 2016; Andrejiova et al., 2016 and SantosVázquez et al., 2016) and showed positive effects on antioxidant activity, productivity and yield.
Yeast extract is the natural compound contains many compounds as proteins and cytokinins that enhance cell enlargement and cell division which are safe and nonpollutant. Also, it contains haloes-6-phosphate synthase which affects plant development (Amer, 2004).

Nanotechnology opens a large scope of novel application in the fields of agricultural industries and biotechnology, because nanoparticles have unique physicochemical properties as, high reactivity, high surface area, particle morphology and tunable pore size. Lithovit is a natural $\mathrm{CO}_{2}$ nano fertilizer contains organic calcite carbonate from natural limestone deposits, suitable and recommended for use in organic farming in the European Community, harmless to humans and animals and not hazardous to water (Bilal, 2010).

This study was carried out to evaluate possible effects of irrigation with magnetized water and some foliar applications on plant growth as well as chemical composition of leaves of tomato plants.

\section{MATERIALS AND METHODS}

Theis experiment was conducted in the two successive summer seasons of 2016 and 2017 on tomato plants "6112" hybride at a private farm at Sahragt ElSoghra near Mansoura, Dakahlia Governorate, Egypt to evaluate tomato plants performance (vegetative characteristics and chemical constituents of leaves) in response to irrigation water treatments (magnetized and non-magnetized), foliar applications (chitosan, lithovit, selenium and yeast extract) and their interactions.

The experiment layout was split plot system in a randomized complete block design with three replicates. The main plots were for irrigation water treatments, while foliar application treatments were distributed in the sub plots. The experimental unit area was $64 \mathrm{~m}^{2}(2$ drip lines $\times 20 \mathrm{~m}$ long $\times 1.6 \mathrm{~m}$ width). The seedlings were transplanted on one side of drip line at $50 \mathrm{~cm}$ apart.

The experiment included 18 treatments which were arranged as water irrigation treatments (Irrigation with magnetized and non-magnetized water) and nine 
foliar application treatments; chitosan (250 and 500 ppm), Lithovit (1.5 and $2 \mathrm{~g} / \mathrm{L})$, selenium as Sodium Selenite (5 and $10 \mathrm{ppm})$, yeast extract ( 5 and $10 \mathrm{~g} / \mathrm{L}$ ) and the control treatment. All foliar spraying solutions were applied three times within 10 days intervals starting from 30 days after transplanting.

Seedlings of 45 days old were transplanted into open field in February $\left(15^{\text {th }}\right.$ and $5^{\text {th }}$ in the first and second season, respectively). During the two growing seasons preparation of the experimental soil, fertigation and pest control were applied as recommended by Egyptian Ministry of Agriculture and land reclamation.

\section{Data recorded}

Microbiological status:

It was evaluated in both soil irrigated with magnetized and untreated water in the second season after 98 days from transplanting according to the method described by Seeley and Van Demark (1981) and shown in Table 1.

Soil status:

Some soil properties were determined in both soil irrigated with magnetized and untreated water in the second season after 103 days from transplanting and shown in Table 2.

Table 1. Types and names of some bacteria presented in the experimental soil.

\begin{tabular}{lcc}
\hline Sample type & Bacteria & Population \\
\hline $\begin{array}{l}\text { Irrigation with non - } \\
\text { magnetized water }\end{array}$ & E. coli & $1 \times 10^{5}$ cells/g dry \\
\hline Bacillus sp. & soil \\
\hline $\begin{array}{l}\text { Irrigation with } \\
\text { magnetized water }\end{array}$ & $\begin{array}{c}\text { Bacillus } s p . \\
\text { Enterobacter } s p .\end{array}$ & $2 \times 10^{6}$ cells/g dry \\
& Klebsiella sp. & soil \\
\hline
\end{tabular}

Table 2. Some soil properties of the experimental soil during the second season:

No. H.W pH EC Anions(meq/L) Cations (meq/L)

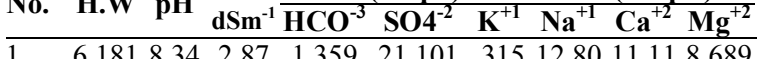

\begin{tabular}{llllllllll}
1 & 6.181 & 8.34 & 2.87 & 1.359 & 21.101 & .315 & 12.80 & 11.11 & 8.689 \\
2 & 6.231 & 8.52 & 1.78 & 1.456 & 12.184 & .197 & 8.542 & 6.130 & 4.130 \\
\hline
\end{tabular}

1: soil irrigated with untreated water.

2: soil irrigated with magnetized water.

Vegetative growth parameters:

Three plants were randomly taken from each treatment after 75 days from transplanting in the two seasons for measuring growth characters of tomato plants as plant height, number of branches/plant, number of leaves/plant, total leaf area/plant according to Koller (1972), fresh and dry weights.

Chemical constituents of leaves:

All studied chemical constituents parameters in tomato leaves were determined at 75 days after transplanting during both seasons. Chlorophylls a, b, total chlorophyll and carotenoids were determined according to the methods described by Wettstein (1957). In addition, nitrogen was determined according to piper (1947). Phosphorus was determined according to the method of Sandell (1950). Potassium was determined by the method described by Horneck and Hanson (1998). Calcium and magnesium were determined according to Jackson (1967). Iron, zinc and manganese were determined according to AOAC (1990).

\section{Statistical analysis:}

The obtained results were subjected to statistical analysis of variance according to Snedecor and Cochran (1967). The treatment means were compared using LSD test as described by Gomez and Gomez (1984).

\section{RESULTS}

\section{Vegetative growth parameters}

Effects of magnetic treatment of irrigation water: Data presented in Table 3 show growth performance of tomato plants in response to irrigation with magnetized and non-magnetized water in the two seasons of study. The obtained results show that growth attributes like plant height, number of branches and leaves, fresh and dry weights as well as total leaf area differed significantly due to irrigation water treatments. Plants irrigated with magnetized water significantly recorded the highest values of all the aforementioned characteristics in both growing seasons as compared with those irrigated with untreated water.

\section{Effects of foliar applications:}

Comparing the effect of foliar application treatments (chitosan, lithovit, yeast extract and selenium) on vegetative growth characteristics of tomato plants, it was found that all vegetative growth parameters increased in response to foliar application treatments in the two growing seasons as compared to the check treatment (sprayed with tap water). Data in Table 3 clearly indicate that higher significant values of all studied parameters were recorded with spraying chitosan at $250 \mathrm{ppm}$ followed by spraying yeast extract at $10 \mathrm{~g} / \mathrm{L}$ in both seasons. Foliar application of lithovit at $1.5 \mathrm{~g} / \mathrm{L}$ came in the third order followed by other foliar application treatments. The control plants recorded the lowest values of all studied vegetative parameters in both seasons.

Effect of interaction between irrigation water treatments and foliar applications:

Data in Table 3 show that plant height, number of branches and leaves, fresh and dry weights and leaf area per plant significantly affected by the interaction between irrigation water and foliar application treatments (chitosan, lithovit, selenium and yeast extract) in the two growing seasons. Higher values of all the aforementioned characters were recorded by plants irrigated with magnetized water and sprayed with all foliar application treatments. Foliar application of chitosan at $250 \mathrm{ppm}$ came in the first order followed by yeast extract at $10 \mathrm{~g} / \mathrm{L}$ then lithovit at $1.5 \mathrm{~g} / \mathrm{L}$. On the other hand, the less values were recorded when plants irrigated with non-magnetized water and sprayed with tap water in the two growing seasons.

\section{Chemical constituents of leaves}

Effects of magnetic treatment of irrigation water:

Data presented in Tables 4 and 5 show the impact of irrigation with magnetized and non-magnetized water on leaf minerals content (N, P, K, Ca, Mg, Fe, Zn and Mn) and pigments (Chl. a, Chl. b, total chlorophyll and carotenoids) of tomato leaves. It was noticed higher significant values of all mentioned parameters were obtained by irrigation with magnetized water except for $\mathrm{Fe}$ in both seasons compared to irrigation with untreated water. Fe content recorded the highest values when plants irrigated with non-magnetized water in both seasons. 
Table 3. Vegetative growth characteristics of tomato plants as affected by irrigation water treatments and foliar applications during summer seasons of 2016 and 2017 after 75 days from transplanting.

\begin{tabular}{|c|c|c|c|c|c|c|c|c|}
\hline \multirow{2}{*}{\multicolumn{3}{|c|}{ Treatments }} & \multicolumn{2}{|c|}{ Plant height(cm) } & \multicolumn{2}{|c|}{ No. of leaves/plant } & \multicolumn{2}{|c|}{ No. of branches /plant } \\
\hline & & & 2016 & 2017 & 2016 & 2017 & 2016 & 2017 \\
\hline \multicolumn{9}{|c|}{ A) Irrigation water treatments: } \\
\hline \multicolumn{3}{|c|}{ Magnetized } & 64.7 & 72.8 & 88.2 & 93.4 & 14.6 & 16.5 \\
\hline \multicolumn{3}{|c|}{ Non-magnetized } & 49.0 & 56.9 & 63.2 & 68.4 & 10.3 & 12.2 \\
\hline \multicolumn{3}{|c|}{ F. test } & $*$ & $*$ & $*$ & $*$ & $*$ & $*$ \\
\hline \multicolumn{9}{|c|}{ B) Foliar applications: } \\
\hline \multirow{2}{*}{\multicolumn{2}{|c|}{ Chitosan }} & $250 \mathrm{ppm}$ & 66.4 & 76.0 & 89.5 & 97.7 & 15.9 & 18.1 \\
\hline & & $500 \mathrm{ppm}$ & 53.5 & 61.4 & 75.1 & 80.3 & 12.1 & 14.4 \\
\hline \multirow{2}{*}{\multicolumn{2}{|c|}{ Lithovit }} & $1.5 \mathrm{~g}$ & 62.1 & 70.0 & 79.8 & 87.9 & 13.0 & 14.9 \\
\hline & & $2 \mathrm{~g}$ & 55.0 & 62.9 & 77.1 & 82.3 & 11.2 & 13.1 \\
\hline \multirow{2}{*}{\multicolumn{2}{|c|}{ Selenium }} & $5 \mathrm{ppm}$ & 55.0 & 62.9 & 76.4 & 81.6 & 12.2 & 14.1 \\
\hline & & $10 \mathrm{ppm}$ & 59.9 & 67.8 & 78.7 & 79.0 & 11.5 & 13.1 \\
\hline \multirow{2}{*}{\multicolumn{2}{|c|}{ Yeast extract }} & $5 \mathrm{~g}$ & 50.0 & 57.8 & 64.3 & 69.5 & 11.0 & 12.9 \\
\hline & & $10 \mathrm{~g}$ & 64.3 & 72.1 & 83.8 & 88.5 & 15.6 & 16.7 \\
\hline \multicolumn{2}{|c|}{ Control } & & 45.7 & 53.0 & 56.5 & 61.3 & 9.7 & 11.6 \\
\hline \multicolumn{3}{|c|}{ L.S.D. at $5 \%$} & 2.1 & 2.3 & 4.6 & 4.3 & 0.5 & 0.6 \\
\hline \multicolumn{9}{|c|}{ Interaction between irrigation water treatments and foliar applications $(\mathrm{A} \times \mathrm{B})$ : } \\
\hline & Chitocan & $250 \mathrm{ppm}$ & 80 & 91.2 & 106.4 & 115.0 & 20.2 & 22.8 \\
\hline \multirow{8}{*}{ 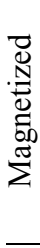 } & Chitosan & $500 \mathrm{ppm}$ & 58.3 & 66.2 & 84.3 & 89.5 & 13.7 & 15.6 \\
\hline & & $1.5 \mathrm{~g}$ & 71.4 & 79.3 & 94.1 & 98.8 & 15.2 & 17.1 \\
\hline & Lithovit & $2 \mathrm{~g}$ & 57.4 & 65.3 & 91.7 & 96.9 & 12.2 & 14.1 \\
\hline & Selenium & $5 \mathrm{ppm}$ & 59.0 & 66.9 & 81.9 & 87.1 & 14.4 & 16.3 \\
\hline & Selenıum & $10 \mathrm{ppm}$ & 69.3 & 77.2 & 84.1 & 89.3 & 12.2 & 13.5 \\
\hline & Yeast extract & $5 \mathrm{~g}$ & 55.8 & 63.6 & 78.8 & 84.0 & 12.6 & 14.5 \\
\hline & Y east extract & $10 \mathrm{~g}$ & 75.9 & 83.6 & 95.9 & 99.3 & 19.8 & 21.0 \\
\hline & Control & & 55.7 & 62.4 & 76.2 & 80.7 & 11.6 & 13.5 \\
\hline \multirow{8}{*}{ 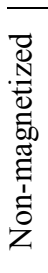 } & Chitosan & $250 \mathrm{ppm}$ & 52.9 & 60.8 & 72.5 & 80.4 & 11.6 & 13.5 \\
\hline & Chitosan & $500 \mathrm{ppm}$ & 48.7 & 56.6 & 65.9 & 71.1 & 10.5 & 12.4 \\
\hline & Lithovit & $1.5 \mathrm{~g}$ & 52.8 & 60.7 & 71.8 & 77.0 & 10.8 & 12.7 \\
\hline & & $2 \mathrm{~g}$ & 52.7 & 60.6 & 62.5 & 67.7 & 10.2 & 12.1 \\
\hline & Selenium & $5 \mathrm{ppm}$ & 51.0 & 58.9 & 70.9 & 76.1 & 10.1 & 12.0 \\
\hline & & $10 \mathrm{ppm}$ & 50.5 & 58.4 & 63.4 & 68.6 & 10.8 & 12.7 \\
\hline & Yeast extract & $5 \mathrm{~g}$ & 44.2 & 52.1 & 49.8 & 55.0 & 9.5 & 11.4 \\
\hline & Control & $10 \mathrm{~g}$ & 52.7 & 60.6 & 75.5 & 77.7 & 11.3 & 13.2 \\
\hline \multicolumn{3}{|c|}{ L.S.D. at $5 \%$} & $\frac{55.8}{2.9}$ & $\frac{43.1}{3.3}$ & $\frac{36.8}{6.5}$ & $\frac{42.0}{62}$ & $\frac{7.8}{0.7}$ & $\frac{9.7}{0.8}$ \\
\hline
\end{tabular}

\begin{tabular}{|c|c|c|c|c|c|c|c|c|}
\hline \multirow{2}{*}{\multicolumn{3}{|c|}{ Treatments }} & \multicolumn{6}{|c|}{ Fresh weight (gm/plant) Dry weight (gm/plant) af area $\left(\mathrm{m}^{2} / \mathrm{plant}\right)$} \\
\hline & & & 2016 & 2017 & 2016 & 2017 & 2016 & 2017 \\
\hline \multicolumn{9}{|c|}{ A) Irrigation water treatments: } \\
\hline \multirow{2}{*}{\multicolumn{3}{|c|}{$\begin{array}{l}\text { Magnetized } \\
\text { Non-magnetized }\end{array}$}} & 817.9 & 868.1 & 90.1 & 95.1 & 22.82 & 24.95 \\
\hline & & & 456.6 & 507.2 & 53.8 & 58.8 & 10.82 & 13.62 \\
\hline \multicolumn{3}{|c|}{ F. test } & & & & & & \\
\hline \multicolumn{9}{|c|}{ B) Foliar applications: } \\
\hline \multirow{2}{*}{\multicolumn{2}{|c|}{ Chitosan }} & $250 \mathrm{ppm}$ & 766.3 & 825.2 & 90.3 & 96.8 & 25.03 & 26.64 \\
\hline & & $500 \mathrm{ppm}$ & 619.0 & 669.5 & 65.9 & 72.9 & 16.93 & 19.21 \\
\hline \multirow{2}{*}{\multicolumn{2}{|c|}{ Lithovit }} & $1.5 \mathrm{~g}$ & 660.8 & 711.4 & 81.6 & 86.6 & 19.53 & 20.98 \\
\hline & & $2 \mathrm{~g}$ & 599.0 & 660.6 & 69.4 & 74.4 & 14.68 & 16.63 \\
\hline \multirow{2}{*}{\multicolumn{2}{|c|}{ Selenium }} & $5 \mathrm{ppm}$ & 625.4 & 676.0 & 69.2 & 74.2 & 12.64 & 14.92 \\
\hline & & $10 \mathrm{ppm}$ & 608.7 & 646.0 & 67.3 & 69.9 & 16.72 & 19.33 \\
\hline \multirow{2}{*}{\multicolumn{2}{|c|}{ Yeast extract }} & $5 \mathrm{~g}$ & 631.5 & 682.0 & 69.6 & 74.2 & 12.79 & 19.07 \\
\hline & & $10 \mathrm{~g}$ & 695.5 & 746.0 & 82.7 & 87.0 & 20.94 & 23.22 \\
\hline \multirow{2}{*}{\multicolumn{2}{|c|}{$\begin{array}{l}\text { Control } \\
\text { L.S.D. at } 5 \%\end{array}$}} & & 529.1 & 572.1 & 51.6 & 56.5 & 12.13 & 13.57 \\
\hline & & & 19.9 & 19.1 & 1.2 & 1.3 & 1.09 & 0.99 \\
\hline \multicolumn{9}{|c|}{ Interaction between irrigation water treatments and foliar applications } \\
\hline \multirow{9}{*}{ 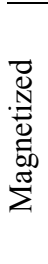 } & 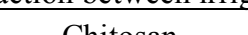 & $250 \mathrm{ppm}$ & 982.3 & 1049.6 & 118.1 & 124.8 & 35.55 & 36.49 \\
\hline & Chitosan & $500 \mathrm{ppm}$ & 806.8 & 857.4 & 85.5 & 94.6 & 23.42 & 25.70 \\
\hline & J ithovit & $1.5 \mathrm{~g}$ & 821.0 & 871.5 & 102.0 & 107.0 & 26.35 & 28.63 \\
\hline & Lithovit & $2 \mathrm{~g}$ & 818.6 & 869.1 & 78.8 & 83.7 & 16.74 & 18.35 \\
\hline & Solonium & $5 \mathrm{ppm}$ & 776.0 & 826.5 & 80.8 & 85.8 & 16.11 & 18.39 \\
\hline & Selenium & $10 \mathrm{ppm}$ & 816.3 & 856.9 & 89.7 & 89.8 & 25.04 & 27.99 \\
\hline & Yeost outroct & $5 \mathrm{~g}$ & 784.4 & 834.9 & 82.1 & 86.4 & 18.61 & 20.89 \\
\hline & Yeast extract & $10 \mathrm{~g}$ & 882.4 & 933.0 & 101.6 & 106.6 & 28.40 & 30.63 \\
\hline & Control & & 673.6 & 714.1 & 72.6 & 77.6 & 15.14 & 17.42 \\
\hline \multirow{10}{*}{ 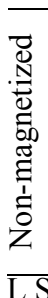 } & Chitosan & $250 \mathrm{ppm}$ & 550.2 & 600.8 & 63.8 & 68.8 & 14.51 & 16.79 \\
\hline & Chitosan & $500 \mathrm{ppm}$ & 431.1 & 481.7 & 46.3 & 51.3 & 10.43 & 12.71 \\
\hline & Iithovit & $1.5 \mathrm{~g}$ & 500.7 & 551.2 & 61.2 & 66.2 & 12.72 & 13.33 \\
\hline & Lithovit & $2 \mathrm{~g}$ & 379.4 & 452.0 & 60.0 & 65.0 & 12.63 & 14.91 \\
\hline & Selenium & $5 \mathrm{ppm}$ & 474.9 & 525.5 & 57.7 & 62.7 & 9.17 & 11.45 \\
\hline & & $10 \mathrm{ppm}$ & 401.4 & 435.2 & 45.0 & 49.9 & 8.40 & 10.68 \\
\hline & & $5 \mathrm{~g}$ & 478.5 & 529.1 & 57.1 & 62.1 & 9.11 & 17.25 \\
\hline & Yeast extract & $10 \mathrm{~g}$ & 508.5 & 559.1 & 62.4 & 67.4 & 13.48 & 15.79 \\
\hline & Control & & 384.6 & 430.0 & 30.5 & 35.5 & 6.97 & 9.73 \\
\hline & . at $5 \%$ & & 28.2 & 27.2 & 1.6 & 1.9 & 1.54 & 1.40 \\
\hline
\end{tabular}


Dawa, K. K. et al.

Table 4. Mineral contents of tomato leaves as affected by irrigation water treatments and foliar applications during summer seasons of 2016 and 2017 after 75 days from transplanting.

\begin{tabular}{|c|c|c|c|c|c|c|c|c|c|c|}
\hline \multirow{2}{*}{\multicolumn{3}{|c|}{ Treatments }} & \multicolumn{2}{|c|}{$\mathbf{N}(\%)$} & \multicolumn{2}{|c|}{$\mathbf{P}(\%)$} & \multicolumn{2}{|c|}{$\mathrm{K}(\%)$} & \multicolumn{2}{|c|}{ Ca (\%) } \\
\hline & & & 2016 & 2017 & 2016 & 2017 & 2016 & 2017 & 2016 & 2017 \\
\hline \multicolumn{10}{|c|}{ A) Irrigation water treatments: } & 0219 \\
\hline \multicolumn{3}{|c|}{ Magnetized. } & 2.36 & 2.77 & 0.246 & 0.288 & 1.54 & 1.03 & 0.103 & 0.2116 \\
\hline & st & & & & & $*$ & & $*$ & & \\
\hline & Foliar applicatio & & & & & & & & & \\
\hline & & $250 \mathrm{ppm}$ & 4.24 & 4.65 & 0.630 & 0.672 & 2.95 & 2.44 & 0.199 & 0.213 \\
\hline & Jsall & $500 \mathrm{ppm}$ & 2.80 & 3.21 & 0.341 & 0.383 & 2.15 & 1.64 & 0.150 & 0.161 \\
\hline Lit & ovit & $1.5 \mathrm{~g}$ & 3.21 & 3.62 & 0.412 & 0.454 & 2.62 & 2.11 & 0.161 & 0.172 \\
\hline & JVIl & $2 \mathrm{~g}$ & 2.87 & 3.28 & 0.349 & 0.391 & 2.21 & 1.70 & 0.145 & 0.161 \\
\hline & & $5 \mathrm{ppm}$ & 3.04 & 3.45 & 0.339 & 0.383 & 2.01 & 1.50 & 0.145 & 0.156 \\
\hline & nium & $10 \mathrm{ppm}$ & 2.78 & 3.19 & 0.363 & 0.405 & 2.37 & 1.87 & 0.152 & 0.163 \\
\hline & t extract & $5 \mathrm{~g}$ & 2.68 & 3.09 & 0.362 & 0.404 & 1.92 & 1.41 & 0.157 & 0.168 \\
\hline & textiat & $10 \mathrm{~g}$ & 3.29 & 3.70 & 0.495 & 0.537 & 2.68 & 2.17 & 0.166 & 0.177 \\
\hline $\mathrm{Co}$ & & & 2.07 & 2.48 & 0.221 & 0.263 & 1.33 & 0.82 & 0.122 & 0.133 \\
\hline L.S & . at $5 \%$ & & 0.28 & 0.28 & 0.047 & 0.047 & 0.23 & 0.23 & 0.007 & 0.008 \\
\hline Int & ction between in & gation water & reatmen & and fo & ir applic & tions $(\mathrm{A}$ & B): & & & \\
\hline & Chitosan & $250 \mathrm{ppm}$ & 5.66 & 6.07 & 0.965 & 1.007 & 3.85 & 3.34 & 0.271 & 0.282 \\
\hline & Cmiosan & $500 \mathrm{ppm}$ & 3.02 & 3.43 & 0.408 & 0.450 & 3.12 & 2.61 & 0.204 & 0.215 \\
\hline 8 & Lithovit & $1.5 \mathrm{~g}$ & 3.82 & 4.23 & 0.540 & 0.582 & 3.37 & 2.86 & 0.209 & 0.221 \\
\hline$\stackrel{N}{Z}$ & Lithovil & $2 \mathrm{~g}$ & 3.23 & 3.64 & 0.464 & 0.506 & 2.70 & 2.19 & 0.201 & 0.212 \\
\hline$\Phi$ & Selenium & $5 \mathrm{ppm}$ & 3.43 & 3.84 & 0.447 & 0.489 & 2.55 & 2.04 & 0.193 & 0.204 \\
\hline ప్ర & seremum & $10 \mathrm{ppm}$ & 3.24 & 3.65 & 0.482 & 0.524 & 3.29 & 2.78 & 0.207 & 0.218 \\
\hline & Yeast extract & $5 \mathrm{~g}$ & 3.35 & 3.76 & 0.498 & 0.540 & 2.26 & 1.75 & 0.209 & 0.220 \\
\hline & Teast exuact & $10 \mathrm{~g}$ & 3.94 & 4.35 & 0.699 & 0.741 & 3.42 & 2.91 & 0.214 & 0.225 \\
\hline & Control & & 2.99 & 3.40 & 0.306 & 0.348 & 2.07 & 1.56 & 0.160 & 0.171 \\
\hline & Chitosan & $250 \mathrm{ppm}$ & 2.82 & 3.23 & 0.295 & 0.337 & 2.05 & 1.54 & 0.126 & 0.143 \\
\hline $\mathbb{N}$ & & $500 \mathrm{ppm}$ & 2.59 & 3.00 & 0.274 & 0.316 & 1.19 & 0.68 & 0.096 & 0.107 \\
\hline$\stackrel{\nabla}{0}$ & Lithovit & $1.5 \mathrm{~g}$ & 2.64 & 3.05 & 0.285 & 0.327 & 1.87 & 1.36 & 0.113 & 0.124 \\
\hline 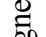 & Lithovit & $2 \mathrm{~g}$ & 2.51 & 2.92 & 0.235 & 0.277 & 1.71 & 1.20 & 0.089 & 0.111 \\
\hline$\underset{\Xi}{\infty}$ & Selenium & 5 ppm & 2.60 & 3.01 & 0.231 & 0.274 & 1.46 & 0.95 & 0.098 & 0.109 \\
\hline$\Xi$ & serenum & $10 \mathrm{ppm}$ & 2.32 & 2.73 & 0.244 & 0.286 & 1.46 & 0.95 & 0.098 & 0.109 \\
\hline อี & Yeast extract & $5 \mathrm{~g}$ & 2.02 & 2.43 & 0.225 & 0.267 & 1.58 & 1.07 & 0.106 & 0.117 \\
\hline & Yeast extract & $10 \mathrm{~g}$ & 2.64 & 3.05 & 0.291 & 0.333 & 1.94 & 1.43 & 0.118 & 0.129 \\
\hline & Control & & 1.16 & 1.57 & 0.136 & 0.178 & 0.60 & 0.09 & 0.085 & 0.096 \\
\hline L.S & D. at $5 \%$ & & 0.40 & 0.40 & 0.067 & 0.067 & 0.330 & 0.331 & 0.010 & 0.011 \\
\hline & itments & & Mg & $\%$ & $\mathrm{Fe}_{\mathrm{J}}$ & pm & $\mathrm{Zn}$ & & MI & pm \\
\hline 10 & tments & & 2016 & 2017 & 2016 & 2017 & 2016 & 2017 & 2016 & 2017 \\
\hline & Irrigation water $t$ & eatments: & & & & & & & & \\
\hline$\overline{\mathrm{Ma}}$ & netized & & 0.558 & 0.546 & 182.2 & 181.1 & 11.937 & 13.278 & 192.15 & 182.06 \\
\hline No & magnetized & & 0.162 & 0.149 & 372.4 & 339.6 & 7.466 & 8.807 & 107.08 & 97.00 \\
\hline F. $t$ & & & & $*$ & $*$ & $*$ & & $*$ & $*$ & $*$ \\
\hline & Foliar applicatio & & & & & & & & & \\
\hline $\mathrm{Ch}$ & osan & $250 \mathrm{ppm}$ & 0.497 & 0.483 & 308.2 & 283.5 & 13.280 & 14.620 & 187.11 & 177.01 \\
\hline & dsall & $500 \mathrm{ppm}$ & 0.339 & 0.326 & 275.4 & 264.2 & 9.115 & 10.456 & 131.83 & 121.73 \\
\hline Lit & ovit & $1.5 \mathrm{~g}$ & 0.392 & 0.380 & 304.2 & 279.8 & 9.775 & 11.115 & 170.03 & 159.98 \\
\hline & & $2 \mathrm{~g}$ & 0.373 & 0.361 & 269.7 & 263.1 & 9.140 & 10.481 & 137.95 & 127.90 \\
\hline & aium & $5 \mathrm{ppm}$ & 0.342 & 0.330 & 246.4 & 236.2 & 8.962 & 10.303 & 142.53 & 132.43 \\
\hline & IntIII & $10 \mathrm{ppm}$ & 0.354 & 0.342 & 273.7 & 252.1 & 9.901 & 10.242 & 145.81 & 135.71 \\
\hline & & $5 \mathrm{~g}$ & 0.361 & 0.349 & 265.8 & 255.8 & 9.340 & 10.681 & 147.28 & 137.18 \\
\hline & t extract & $10 \mathrm{~g}$ & 0.408 & 0.394 & 389.3 & 395.6 & 11.256 & 12.597 & 178.63 & 168.53 \\
\hline $\mathrm{Co}$ & & & 0.173 & 0.161 & 162.8 & 113.0 & 7.547 & 8.887 & 105.40 & 95.30 \\
\hline L.S & . at $5 \%$ & & 0.063 & 0.063 & 26.31 & 44.07 & 0.994 & 0.993 & 5.870 & 5.864 \\
\hline & action between & ation water & reatmen & and fo & Ir applic & tions $(\mathrm{A}$ & B): & & & \\
\hline & & $250 \mathrm{ppm}$ & 0.794 & 0.782 & 167.7 & 204.7 & 17.191 & 18.529 & 244.20 & 234.10 \\
\hline & & $500 \mathrm{ppm}$ & 0.565 & 0.553 & 182.2 & 172.2 & 11.284 & 12.625 & 166.23 & 156.13 \\
\hline 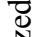 & Lithovit & $1.5 \mathrm{~g}$ & 0.604 & 0.592 & 208.7 & 200.8 & 11.492 & 12.833 & 214.63 & 204.53 \\
\hline$\underset{7}{Z}$ & & $2 \mathrm{~g}$ & 0.572 & 0.560 & 199.8 & 189.8 & 11.085 & 12.426 & 166.23 & 156.23 \\
\hline Ф & Selenium & $5 \mathrm{ppm}$ & 0.516 & 0.504 & 194.1 & 184.1 & 11.313 & 12.654 & 187.63 & 177.53 \\
\hline ED & serentim & $10 \mathrm{ppm}$ & 0.533 & 0.521 & 210.8 & 199.1 & 10.728 & 12.069 & 149.20 & 184.10 \\
\hline$\Sigma$ & Yeast extract & $5 \mathrm{~g}$ & 0.576 & 0.564 & 183.4 & 173.4 & 11.207 & 12.548 & 178.43 & 168.33 \\
\hline & reast exuract & $10 \mathrm{~g}$ & 0.616 & 0.604 & 214.7 & 237.3 & 13.270 & 14.611 & 231.60 & 221.50 \\
\hline & Control & & 0.247 & 0.235 & 78.40 & & 9.864 & 11.205 & 146.23 & 136.13 \\
\hline & Chito & $250 \mathrm{ppm}$ & 0.201 & 0.185 & 405.6 & 362.2 & 9.369 & 10.710 & 130.03 & 119.93 \\
\hline 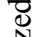 & Cnitosan & $500 \mathrm{ppm}$ & 0.113 & 0.099 & 368.7 & 356.3 & 6.947 & 8.288 & 97.43 & 87.33 \\
\hline 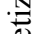 & Lithovit & $1.5 \mathrm{~g}$ & 0.181 & 0.168 & 399.6 & & 8.057 & 9.398 & 125.43 & 115.43 \\
\hline 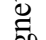 & Litnovit & $2 \mathrm{~g}$ & 0.174 & 0.161 & 339.7 & 336.4 & 7.195 & 8.536 & 109.66 & 99.56 \\
\hline$\underbrace{\infty}$ & Selenium & $5 \mathrm{ppm}$ & 0.169 & 0.157 & 298.7 & 288.3 & 6.611 & 7.952 & 97.43 & 87.33 \\
\hline$\Xi$ & SeTEIIIUII & $10 \mathrm{ppm}$ & 0.175 & 0.163 & 379.7 & 305.0 & 7.075 & 8.416 & 97.43 & 87.33 \\
\hline$\stackrel{1}{0}$ & Yeast extract & $5 \mathrm{~g}$ & 0.145 & 0.133 & 348.2 & 338.2 & 7.473 & 8.814 & 116.13 & 106.03 \\
\hline & reast extract & $10 \mathrm{~g}$ & 0.199 & 0.185 & 563.9 & 553.9 & 9.241 & 10.582 & 125.66 & 115.56 \\
\hline & Control & & 0.100 & 0.088 & 247.3 & 157.7 & 5.230 & 6.569 & 64.56 & 54.46 \\
\hline L.S & D. at $5 \%$ & & 0.089 & 0.089 & 37.21 & 62.33 & 1.405 & 1.405 & 8.301 & 8.293 \\
\hline
\end{tabular}


Effects of foliar applications:

The obtained results in Tables 4 and 5 show that all foliar application treatments significantly enhanced minerals and photosynthetic pigments in tomato leaves as compared with check plants in both growing seasons. Chitosan application at the lowest concentration (250 ppm) is the superior in its effects on all studied parameters, followed by decline with the highest concentration $(500 \mathrm{ppm})$ but still higher than the control except for Fe. On the other hand, Foliar application of yeast extract at $10 \mathrm{~g} / \mathrm{L}$ came in the first order in enhancing Fe content followed Chitosan (250 ppm) then lithovit at $1.5 \mathrm{~g} / \mathrm{L}$ in both growing seasons.

Effect of interaction between irrigation water treatments and foliar applications:

Tomato leaf minerals (N, P, K, Ca, Mg, Fe, Zn and $\mathrm{Mn}$ ) and pigments (Ch. a, Ch. b, total chlorophyll and carotenoids) in response to the interaction between irrigation water treatments and foliar applications are presented in Tables 4 and 5. Data indicated that the best values of all the aforementioned parameters significantly enhanced in response to irrigation with magnetized water and foliar applications (chitosan, lithovit, selenium and yeast extract) except for $\mathrm{Fe}$ in both seasons. The highest values of $\mathrm{N}, \mathrm{P}, \mathrm{K}, \mathrm{Ca}, \mathrm{Mg}$, $\mathrm{Zn}, \mathrm{Mn}, \mathrm{Chl}$. a, Chl. b, total chlorophyll and carotenoids were obtained specially with chitosan at $250 \mathrm{ppm}$. On the other hand, both the lowest values of the previous parameters and the best value of Fe were recorded with the control treatment (irrigation with untreated water in the absence of foliar applications) in both seasons.

Table 5. Photosynthetic pigments of tomato leaves as affected by irrigation water treatments and foliar applications during summer seasons of 2016 and 2017 after 75 days from transplanting.

\begin{tabular}{|c|c|c|c|c|c|c|c|c|c|c|}
\hline \multirow{2}{*}{\multicolumn{2}{|c|}{ Treatments }} & & \multicolumn{2}{|c|}{$\begin{array}{c}\text { Chl. (a) } \\
\text { (mg/100gm F.Wt.) }\end{array}$} & \multicolumn{2}{|c|}{$\begin{array}{c}\text { Chl. (b) } \\
\text { (mg/100gm F.Wt.) }\end{array}$} & \multicolumn{2}{|c|}{$\begin{array}{l}\text { Total chl. }(a+b) \\
(\mathrm{mg} / \mathbf{1 0 0 g m} \text { F.Wt. }\end{array}$} & \multicolumn{2}{|c|}{$\begin{array}{c}\text { Carotenoids } \\
\text { (mg/100gm F.Wt.) }\end{array}$} \\
\hline & & & 2016 & 2017 & 2016 & 2017 & 2016 & 2017 & 2016 & 2017 \\
\hline \multicolumn{11}{|c|}{ A) Irrigation water treatments: } \\
\hline \multicolumn{3}{|c|}{ Magnetized } & 1.144 & 1.233 & 0.716 & 0.775 & 1.861 & 2.009 & 0.668 & 0.672 \\
\hline \multicolumn{3}{|c|}{ Non-magnetized } & 0.600 & 0.680 & 0.536 & 0.597 & 1.136 & 1.278 & 0.503 & 0.507 \\
\hline \multicolumn{3}{|c|}{ F. test } & & & & & & & & \\
\hline \multicolumn{11}{|c|}{ B) Foliar applications: } \\
\hline \multirow{2}{*}{\multicolumn{2}{|c|}{ Chitosan }} & $250 \mathrm{ppm}$ & 1.270 & 1.356 & 0.767 & 0.822 & 2.038 & 2.178 & 0.709 & 0.697 \\
\hline & & $500 \mathrm{ppm}$ & 0.795 & 0.890 & 0.547 & 0.600 & 1.343 & 1.491 & 0.570 & 0.581 \\
\hline \multirow{2}{*}{\multicolumn{2}{|c|}{ Lithovit }} & $1.5 \mathrm{~g}$ & 0.953 & 1.034 & 0.657 & 0.737 & 1.610 & 1.772 & 0.605 & 0.617 \\
\hline & & $2 \mathrm{~g}$ & 0.858 & 0.944 & 0.600 & 0.659 & 1.458 & 1.603 & 0.557 & 0.569 \\
\hline \multirow{2}{*}{\multicolumn{2}{|c|}{ Selenium }} & $5 \mathrm{ppm}$ & 0.883 & 0.975 & 0.605 & 0.661 & 1.488 & 1.628 & 0.550 & 0.550 \\
\hline & & $10 \mathrm{ppm}$ & 0.798 & 0.879 & 0.595 & 0.651 & 1.393 & 1.531 & 0.561 & 0.572 \\
\hline \multirow{2}{*}{\multicolumn{2}{|c|}{ Yeast extract }} & $5 \mathrm{~g}$ & 0.793 & 0.873 & 0.622 & 0.685 & 1.415 & 1.559 & 0.575 & 0.587 \\
\hline & & $10 \mathrm{~g}$ & 0.955 & 1.036 & 0.737 & 0.800 & 1.693 & 1.836 & 0.665 & 0.665 \\
\hline \multirow{2}{*}{\multicolumn{2}{|c|}{$\begin{array}{l}\text { Control } \\
\text { L.S.D. at } 5 \%\end{array}$}} & & 0.545 & 0.632 & 0.505 & 0.561 & 1.050 & 1.193 & 0.482 & 0.470 \\
\hline & & & 0.024 & 0.030 & 0.062 & 0.064 & 0.075 & 0.081 & 0.045 & 0.045 \\
\hline \multicolumn{11}{|c|}{ Interaction between irrigation water treatments and foliar applications $(\mathrm{A} \times \mathrm{B})$ : } \\
\hline \multirow{9}{*}{ 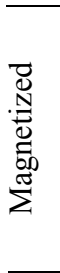 } & Chi & $250 \mathrm{ppm}$ & 1.485 & 1.577 & 0.955 & 0.885 & 2.370 & 2.517 & 0.848 & 0.837 \\
\hline & Chitosan & $500 \mathrm{ppm}$ & 1.075 & 1.185 & 0.704 & 0.620 & 1.695 & 1.857 & 0.637 & 0.648 \\
\hline & I ith it & $1.5 \mathrm{~g}$ & 1.165 & 1.163 & 0.833 & 0.750 & 1.915 & 2.081 & 0.687 & 0.700 \\
\hline & Lithovit & $2 \mathrm{~g}$ & 1.070 & 1.248 & 0.706 & 0.655 & 1.725 & 1.870 & 0.616 & 0.628 \\
\hline & Sele & $5 \mathrm{ppm}$ & 1.145 & 0.243 & 0.724 & 0.670 & 1.815 & 1.958 & 0.637 & 0.625 \\
\hline & Selenıum & $10 \mathrm{ppm}$ & 1.065 & 1.148 & 0.758 & 0.700 & 1.765 & 1.906 & 0.620 & 0.630 \\
\hline & Yeast & $5 \mathrm{~g}$ & 1.135 & 1.216 & 0.736 & 0.685 & 1.820 & 1.952 & 0.628 & 0.640 \\
\hline & extract & $10 \mathrm{~g}$ & 1.170 & 1.251 & 0.940 & 0.880 & 2.050 & 2.206 & 0.773 & 0.785 \\
\hline & Control & & 1.056 & 1.136 & 0.671 & 0.605 & 1.595 & 1.736 & 0.571 & 0.559 \\
\hline \multirow{10}{*}{ 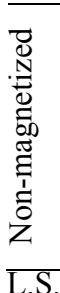 } & Chitosan & $250 \mathrm{ppm}$ & 0.990 & 1.078 & 0.657 & 0.650 & 1.706 & 1.840 & 0.570 & 0.558 \\
\hline & Cnitosan & $500 \mathrm{ppm}$ & 0.516 & 0.596 & 0.529 & 0.475 & 0.991 & 1.125 & 0.503 & 0.515 \\
\hline & I ithovit & $1.5 \mathrm{~g}$ & 0.741 & 0.821 & 0.642 & 0.565 & 1.306 & 1.463 & 0.523 & 0.535 \\
\hline & Litnovit & $2 \mathrm{~g}$ & 0.646 & 0.726 & 0.611 & 0.545 & 1.191 & 1.337 & 0.498 & 0.510 \\
\hline & & $5 \mathrm{ppm}$ & 0.621 & 0.701 & 0.598 & 0.540 & 1.161 & 1.299 & 0.463 & 0.475 \\
\hline & Selenıum & $10 \mathrm{ppm}$ & 0.531 & 0.611 & 0.544 & 0.490 & 1.021 & 1.155 & 0.503 & 0.515 \\
\hline & Yeast & $5 \mathrm{~g}$ & 0.451 & 0.531 & 0.635 & 0.560 & 1.011 & 1.166 & 0.523 & 0.535 \\
\hline & extract & $10 \mathrm{~g}$ & 0.741 & 0.821 & 0.646 & 0.595 & 1.336 & 1.467 & 0.558 & 0.546 \\
\hline & Control & & 0.100 & 0.185 & 0.464 & 0.406 & 0.506 & 0.650 & 0.393 & 0.381 \\
\hline & \multicolumn{2}{|c|}{ L.S.D. at $5 \%$} & 0.034 & 0.42 & 0.090 & 0.088 & 0.107 & 0.115 & 0.064 & 0.064 \\
\hline
\end{tabular}

\section{DISCUSSION}

The stimulating effect of irrigation with magnetized water may be due to improving and increasing Free-living micro-organisms population and activity in soil (Table 1), which in turn enhance root development, increase water and mineral uptake and produce plant hormones that might be responsible for better growth of tomato plants. Also, magnetic treatments enhance the activation of phytohormone and bio-enzyme systems, affects cell membranes structures and in this way increases their permeability and ion, which then affects various metabolic pathway activities as well as increasing the rate of water absorption. Also, there are some changes occurred in the chemical and physical properties of water according to magnetic treatment, mainly hydrogen bonding, surface tension, conductivity, polarity and solubility of salts, these changes in water properties may affec plants growth (Turker et al., 2007and Maheshwari and Grewal, 2009). Changing water and soil properties in response to magnetized water (Table 2) increased the availability and absorption of nutrients causing higher contents of them in plant tissue. Also, 
increasing all photosynthetic pigments may be through the increase in cytokinin synthesis which induced by magnetic field (Atak et al., 2003). Similar enhancing effects were obtained by Abou El-Yazied et al. (2012); Yusuf et al. (2016) and Yusuf and Ogunlela (2017) on tomato plants.

The stimulatings effect of chitosan on plant growth performance (vegetative growth parameter, leaf minerals and pigments content) may be attributed to improving uptake of water and availability of essential nutrients through adjusting cell osmotic pressure and reducing accumulating the harmful free radicals by increasing antioxidants and enzymatic activities (Guan et al., 2009). Also, chitosan enhance key enzymes activity of nitrogen (N) metabolism (glutamine and nitrate reductase) and improve the transportation of nitrogen in the functional leaves as well as increase photosynthesis efficiency which in turn enhance plant growth and development (Mondal et al., 2016). These results are in harmony with the findings of Borkowski et al. (2007); El-Tantawy (2009) and Mondal et al. (2016) on tomato plants.

The increment in the studied parameters that achieved with foliar application of lithovit as compared to the control may be through the role as a long term reservoir supplying plants with $\mathrm{CO}_{2}$, so it can enhance plant growth, where elevated $\mathrm{CO}_{2}$ concentrations generally increased carbon assimilation, photosynthesis intensity, biomass and leaf area of plants (Bilal, 2010 and Maswada and Abd ElRahman, 2014). Also, lithovit consisting mainly of $\mathrm{Ca}$ (3\%), $\mathrm{Mg}(2 \%), \mathrm{CaCo}_{3}$ (24\%) and $\mathrm{MgCo}_{3}$ micron (41\%) which penetrate rapidly into plant tissue and play vital roles in physiological and biological processes in plants in which reflected positively on crop productivity. Similar findings were reported by Byan (2014); Moisa and Berar (2015) and recently Abd El-Nabi et al. (2017).

Concerning the beneficial effect of yeast extract, it is a natural source of cytokinins that stimulate cell enlargement and cell division as well as the synthesis of nucleic acid, protein, and chlorophyll pigments. In addition, yeast extract contains protective agents as sugars, proteins, amino acids, several vitamins as well as most of nutritional elements and organic compounds. The enhanced effects of foliar application of yeast extract on tomato plants were also obtained by Abou ElYazied and Mady (2011); Abd El-Fatah et al. (2014) and Shabana et al. (2015).

Also, exogenous foliar application of $\mathrm{Se}$ has already shown to enhance vegetative growth and chemical constituents of leaves by Abul-Soud and Abd Elrahman (2016) on eggplant; Andrejiova et al. (2016) and Santos-Vázquez et al. (2016) on tomato plants.

\section{REFERENCES}

Abd El-Fatah, A.M.A.; K.K. Dawa, and T.M. Al-Gazzar (2014). Effect of organic fertilizer, biofertilizer and some foliar application treatments on tomato fruit yield and quality (Lycopersicin esculentum Mill). M sc. Fac. Agric., Mans. Univ., Egypt.

Abdel Nabi, H.MA. ; K.K. Dawa ; E.I. El-Gamily and Y.F.E. Imryed (2017). Effect of magnetic water, foliar application with nano material and nitrogen levels on productivity and quality of head lettuce. Int. J. Adv. Res. Biol. Sci., 4(5): 171-181.
Abou El-Yazied A.; A.M. El-Gizawy; S.M. Khalf; A. El-Satar and O.A. Shalaby (2012). Effect of magnetic field treatments for seeds and irrigation water as well as $\mathrm{N}, \mathrm{P}$ and $\mathrm{K}$ levels on productivity of tomato plants. J. App. Sci. Res., 8(4):2088-2099.

Abou El-Yazied, A. and A.M. Mady (2011). Effect of Naphthalene Acetic Acid and Yeast Extract on Growth and Productivity of Tomato (Lycopersicon esculentum Mill.) Plants. Res. J. Agric. and Biol. Sci., 7(2): 271-281.

Abul-Soud, M.A. and H. Abd-Elrahman (2016). Foliar Selenium Application to Improve the Tolerance of Eggplant Grown under Salt Stress Conditions. International J. Plant and Soil Sci., 9(1): 1-10.

Amer, S.S.A. (2004). Growth, green pods yield and seeds yield of common bean (Phaseolus vulgaris L) as affected by active dry yeast, Salicylic acid and their interaction. J. Agric. Sci. Mansoura. Univ., 29(3): 1407-1422.

Andrejiova, A.; A. Hegedusova and I. Mezeyoya (2016). Effect of genotype and selenium biofortification on content of important bioactive substances in tomato (Lycopersicon esculentum Mill). J. International . Sci. Publications, 4: 8-18.

AOAC. (1990). Official Method of Analysis, $15^{\text {th }}$ Ed., Association of Official Analytical Chemists, Inc., USA.

Atak, C.; O. Emiroglu; S. Aklimanoglu, and A. Rzakoulieva (2003). Stimulation of regeneration by magnetic field in soybean (Glycin $\max \mathrm{L}$. Mirrill) tissu cultures. J. cell and Molecular Biol., 2: 113-119.

Bilal B.A. (2010). Lithovit: An innovative fertilizer. The $3^{\text {rd }}$ e-Conference on Agricultural Biosciences (IeCAB 2010), $1^{\text {st }} 15^{\text {th }}$ June 2010. http://www.slideserve.com/madison/lithovitaninnovative-fertilizer.

Borkowski, J.; B. Dyki; A. Felczyńska and W. J. Kowalczyk (2007). Effect of biochikol 020 pc (chitosan) on the plant growth, fruit yield and healthiness of tomato plant roots and stems. J. Borkowski, B. Dyki, A. Felczyńska, W. Kowalczyk, 217-223.

Byan, U. A.I. (2014). Influence of using some safety materials on water requirement and water use efficiency of snap bean plant. Arab Univ. J. Agric. Sci. Ain Shams Univ., Cairo, 22(2): 381394.

Chibu, H. and H. Shibayama (2003). Effects of chitosan application on the growth of several crops. In: Chitin and chitosan in life science. T.Uragami, K Kurita, and T. Fukamizo (eds.).Yamaguchi, Japan. pp. 235-239.

El-Tantawy, E.M (2009). Behaviour of tomato plants as affected by spraying with chitosan and aminofort as natural stimulator substances under application of soil organic amendments. Pakistan J. Biol. Sci., 12:1164-1173.

Gomez, K.A. and A.A. Gomez (1984). Statistical Procedures for the Agricultural Researches. John Wiley and Son, Inc. New York. 
Guan, Y.J.; J. Hu; X.J. Wang and C.X. Shao (2009). Seed priming with chitosan improves maize germination and seedling growth in relation to physiological changes under low temperature stress. J. Zhejiang Univ. Sci., 10: 427-433.

Horneck, D.A. and D. Hanson (1998). Determination of potassium and sodium by Flame Emission Spectrophotometry. In Handbook of Reference Methods for Plant Analysis, pp. 153-155.

Jackson, M. L. (1967). Soil chemical analysis prentic hallpvt. Itd. India. PP. 398.

Koller, H.R. (1972). Leaf area-Leaf weight relationship in soybean canopy. Crop Sci., 12:180-183.

Maheshwari, B.L. and H.S. Grewal (2009). Magnetic treatment of irrigation water: its effect on vegetable crop yield and water productivity. Agriculture Water Management. 96: 1229-1236.

Maswada H.F. and L.A. Abd El-Rahman (2014). Inducing salinity tolerance in wheat plants by hydrogen peroxide and lithovit "A nano- $\mathrm{CaCO}_{3}$ fertilizer". J. Agric. Res. Kafr El-Sheikh Univ. 40 (4): 696-719.

Moisa, R. and V. Berar (2015). Study of the effect of several natural fertilizers on the seedlings leaf area for some tomato cultivars. J. Horti. Forestry and Biotech., (2): 77- 81.

Mondal, A.M.A.; A.B. Puteh and N.C. Dafader (2016). Foliar application of chitosan improved morphophysiological attributes and yield in summer tomato (Solanum lycopersicum). J. Agri. Sci., 53(2): 339-344.

Piper, C.S. (1947). "Soil and Plant Analysis". The Univ. of Adelaiada, Pp: 293-296.

Sandell, R. (1950). Colorimetric determination of traces of metal $2^{\text {nd }}$ Ed. Interscience pub., Inc. New York.
Santos-Vázquez, M.E.; A.B. Mendoza; N.A.R. Torres; M.C. Fuente and A.M. Moreno (2016). Sodium selenite treatment of vegetable seeds and seedlings and the effect on antioxidant status. Emirates J. of Food and Agric., 28(8): 589-593.

Seeley, H.W. and P.J. VanDemark (1981). Microbes in action. A laboratory manual of microbiology. 3rd Edition W.H Freeman and Company USA., p: 350 .

Shabana, A.I.; Ahmed, H.I.; Shafeek, M. R. and F.S. AbdEl-Al (2015). Improving productivity of tomato crop grown under high temperature condition using some safe and natural substances. Middle East J. Agric. Res., 4(1): 20-26.

Snedecor, G.W. and W. G. Cochran (1967). "Statistical Methods". $7^{\text {th }}$ ed. Lowa State Univ. Press, Ames, Lowa, U.S.A., Pp: 507.

Turker, M., C. Temirci, P. Battal and M.E. Erez (2007). The effects of an artificial and static magnetic field on plant growth, chlorophyll and phytohormone levels in maize and sunflower plants. Phyton Ann. Rei Bot., 46: 271-284.

Wettestein, D. (1957). "Chlorophyll". Lethale und der Submikroskopische Formwechsel der plastiden. Exptl. Cell Reso., 12: 427-506.

Yusuf, K.O. and A.O. Ogunelela (2017). Effects of magnetized water on the vegetative growth and yield of tomato. AgricEngInt: CIGR Journal Open access at http://www.cigrjournal.org. 19(1): $1-8$.

Yusuf, K.O.; A.O. Ogunelela and M.O. Murtala (2016). Effects of magnetically treated water on germination and growth of tomato (Lycopersicon esculentum: variety UC82B) under poor soil fertility and deficit irrigation. J. Res. in Forestry, Wildlife and Environ., 8(4): 30-38.

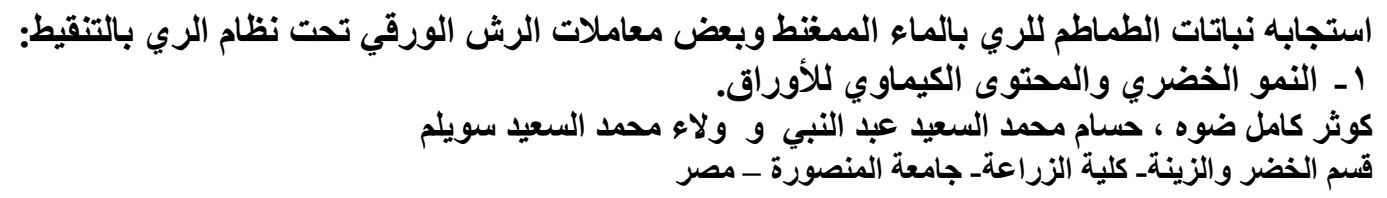

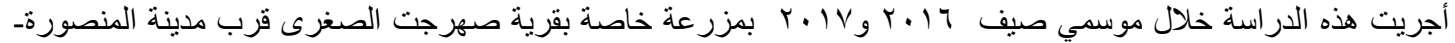

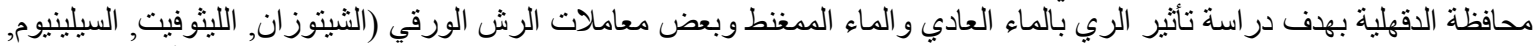

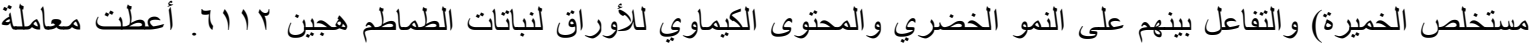
الري بالماء الممغنط أعلى القيم لصفات النمو الخضري والمئلة في ارتفاع النبات, عدد الأوراق, عدد الأفرع, المساحة الورقية, الوزن الطازج والجاف باء وكذلك المحتوى الكيماوي للأوراق مثل كلوروفيل أ, كلوروفيل ب, الكلوفئ الكوروفيل الكلي أ+

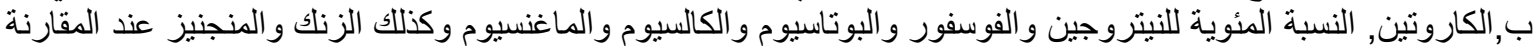

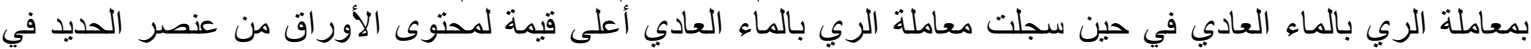

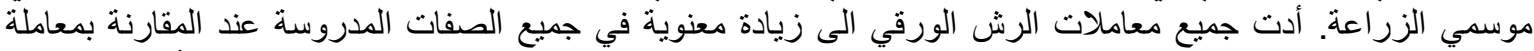

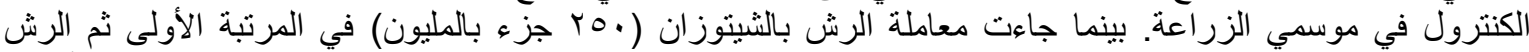

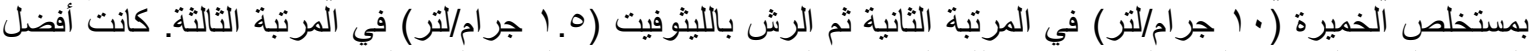
النتائج بالنسبة لصفات النمو الخضري وكذلك المحتوى الكيماوي ناتجة عن الري التربي بالماء الممغنط مع استخدام جميع مواد

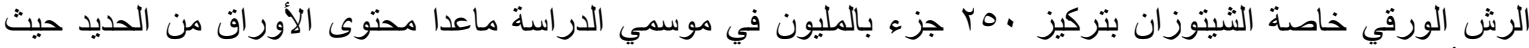
كانت أفضل نتائجه مع الري بالماء العادئ العادي. 